\title{
Cristalização da zeólita ZSM-5 através do método de conversão de gel seco
}

\author{
J. R. SANTOS ${ }^{1}$, F. L. dos SANTOS ${ }^{1}$, A.O.S. da SILVA ${ }^{1}$, S. L. ALENCAR ${ }^{1}$, B. J. B. da \\ SILVA $^{1}$ \\ ${ }^{1}$ Universidade Federal de Alagoas, Faculdade de Engenharia Química \\ E-mail para contato: julyane.rocha@hotmail.com
}

\begin{abstract}
RESUMO - A zeólita ZSM-5 é utilizada como catalisador em reações nas áreas petroquímica, petrolífera e química fina, destacando-se as reações de craqueamento, isomerização, alquilação, aromatização de olefinas, entre outras. Neste trabalho é proposto a síntese da zeólita ZSM-5, através do método de síntese conversão de gel seco, utilizando método conversão assistido de vapor. $\mathrm{Na}$ síntese das amostras de ZSM-5 foram utilizados: pseudobohemita como fonte de alumínio, sílica gel como fonte de silício, hidróxido de sódio como fonte de sódio e como fonte de direcionador estrutural o brometo de tetrapropilamônio (TPABr). Todas as amostras sintetizadas foram caracterizadas por DRX, porém, apenas a amostra utilizada como padrão de referência, sendo a amostra mais cristalina, foi caracterizada também por TG/DTG e BET a fim de compreender suas propriedades físico-químicas. A eficiência dos métodos aplicados no presente trabalho foi comprovada pela estrutura bem definida da zeólita ZSM-5.
\end{abstract}

\section{INTRODUÇÃO}

As zeólitas fazem parte de um amplo grupo de sólidos porosos denominados peneiras moleculares, devido à capacidade de adsorver moléculas compatíveis com as dimensões de seus poros, ao passo que excluem moléculas incompatíveis. Apesar de serem conhecidas em sua forma natural desde o século XVIII, foi somente após a obtenção das primeiras formas sintéticas, em 1930, que as zeólitas deram origem a uma vasta gama de aplicações (Masters e Maschmeyer, 2011). As zeólitas constam de uma rede cristalina formada pela combinação tridimensional de tetraedros $\mathrm{TO}_{4}(\mathrm{~T}=\mathrm{Si}, \mathrm{Al}, \mathrm{B}, \mathrm{Ga}, \mathrm{Ge}, \mathrm{Fe}, \mathrm{P}, \mathrm{Co}$, etc.) unidos entre si através de átomos de oxigênio. A estrutura de uma zeólita apresenta canais e cavidades de dimensões moleculares aos quais se encontram cátions de compensação, moléculas de água, adsorvatos e sais (Giannetto, 1990).

A zeólita ZSM-5 (Zeolite Socony Mobil - 5) foi sintetizada pela primeira vez pelo laboratório da Mobil Corporation. É uma zeólita que se caracteriza pelo alto teor de silício na estrutura (relação Si/Al entre 11 e infinito), pertence ao grupo da família pentasil e possui estrutura tipo MFI (Mobil Five) de acordo com a classificação da International Zeolite Association (IZA). A MFI é uma estrutura cristalina com célula unitária ortorrômbica constituída por 96 tetraedros $\mathrm{TO}_{4}$ (Giannetto, 1990).

Nos últimos anos pesquisadores vem estudando um novo método de síntese de zeólitas, denominado conversão de gel seco (Dry Gel Conversion, DGC). O método conversão de gel seco pode ser dividido em dois tipos: método conversão assistido de vapor (Steam Assisted Conversion, SAC) e método transporte em fase vapor (Vapor Phase Transport, VPT). No 
método de conversão assistido de vapor, o hidrogel em pó sintetizado contém direcionador orgânico estrutural e é fisicamente separado de uma pequena quantidade de água pura localizada no fundo de uma autoclave. O método de transporte em fase vapor é similar ao método de conversão assistido de vapor, exceto que o direcionador orgânico estrutural não está inserido no hidrogel, e sim, em uma solução aquosa no fundo da autoclave (Chen e Huang, 2009).

Diante do exposto, o presente estudo teve como intuito, avaliar a síntese da zeólita ZSM-5, através do método de síntese conversão assistido de vapor, utilizando o brometo de tetrapropilamônio como direcionador estrutural, tão como caracterizar as propriedades físicoquímicas das amostras de ZSM-5 através de diversas técnicas de análises (DRX, TG/DTG e BET).

\section{EXPERIMENTAL}

\subsection{Síntese da ZSM-5 através do método de conversão assistido de vapor}

O gel com a seguinte composição 10,6 TPABr: 14,3 $\mathrm{Na}_{2} \mathrm{O}: 2,0 \quad \mathrm{Al}_{2} \mathrm{O}_{3}: 100 \mathrm{SiO}_{2}: 2000$ $\mathrm{H}_{2} \mathrm{O}$ foi preparado. Para o preparo do gel, dissolveu-se $\mathrm{NaOH}$ (76\% p/p, Merck) em $40 \%$ da água requerida, adiciona-se sulfato de alumínio octadecahidratado (72\% p/p, Sasol) seguido de agitação por 20 minutos, a solução formada denominou-se solução A. Em seguida, preparou-se outra solução denominada de solução B através da dissolução da sílica gel $(96 \%$ p/p, Sigma-Aldrich) em $60 \%$ da água e a adição de brometo de tetrapropilamônio $(98 \%$ p/p, Sigma-Aldrich), seguida de agitação por 20 minutos. Depois do tempo decorrido misturou-se as soluções A e B e manteve-se o sistema sob agitação por mais 30 minutos. Em seguida, o material obtido foi transferido para a estufa para evaporação de toda a água, a temperatura de $60^{\circ} \mathrm{C}$ durante 48 horas. $\mathrm{O}$ material sólido obtido foi triturado em um almofariz e transferido para um suporte de teflon. Baseado nos trabalhos de Matsukata et al. (1999), no fundo da autoclave foi colocado $40 \mathrm{~mL}$ de água destilada. As amostras foram retiradas da estufa em diferentes tempos de cristalização (5 a 11 horas). Após a cristalização, o sólido resultante do processo de cristalização foi separado do líquido sobrenadante por centrifugação (lavado até $\mathrm{pH}$ neutro) e seco em estufa a $120^{\circ} \mathrm{C}$ por 12 horas.

\subsection{Caracterização das amostras}

As amostras foram caracterizadas por difração de raios $X$ (DRX), utilizando um difratômetro da Shimadzu, modelo XRD-6000, com radiação $\mathrm{CuK} \alpha(\lambda=0,1542 \mathrm{~nm})$, filtro de $\mathrm{Ni}$, voltagem de $40 \mathrm{kV}$ e corrente de $30 \mathrm{~mA}$. A aquisição dos dados foi realizada no intervalo de $2 \theta$ entre 3 e $40^{\circ}$, com velocidade de varredura de $2^{\circ} / \mathrm{min}$ e passo de $0,02^{\circ}$. A melhor amostra foi caracterizada também por análise de adsorção/dessorção de nitrogênio e análises térmicas. As análises de adsorção foram realizadas utilizando o equipamento Micromeritics ASAP 2020 com o software versão 3. A amostra foi desgaseificadas por 12 horas, utilizando gás nitrogênio a $77 \mathrm{~K}$, para garantir que o carvão estivesse livre de material orgânico. A adsorção e dessorção do $\mathrm{N}_{2}$ foram realizadas a $350{ }^{\circ} \mathrm{C}$. As análises térmicas foram realizadas numa termobalança DTG-60H, Shimadzu, onde foram utilizados cadinhos de alumina e massas de amostra de aproximadamente $10 \mathrm{mg}$. Utilizou-se uma taxa de aquecimento 10 
${ }^{\circ} \mathrm{C} / \mathrm{min}$, na faixa de temperatura ambiente até $800{ }^{\circ} \mathrm{C}$, em atmosfera dinâmica de ar sintético com vazão de $50 \mathrm{~mL} / \mathrm{min}$.

\section{RESULTADOS E DISCUSSÃO}

\subsection{Difratometria de raios $X$}

Os resultados obtidos foram analisados em comparação ao banco de dados apresentados pela IZA (Internacional Zeolite Association). A Figura 1 abaixo apresenta o difratograma referencial de uma amostra de MFI apresentada pela IZA.

Figura 1 - Difratograma referencial de uma amostra de MFI apresentada pela IZA.

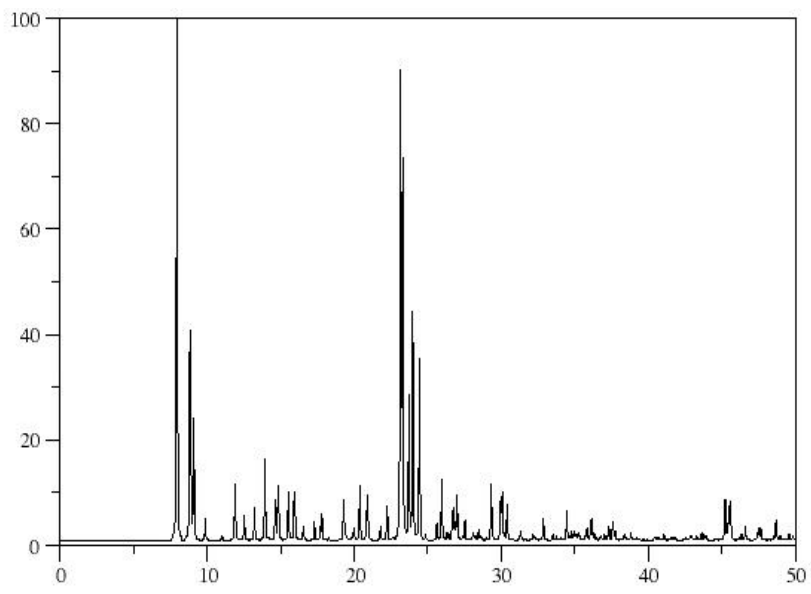

$\mathrm{Na}$ Figura 2, são demonstrados os difratogramas das amostras sintetizadas, onde verificou a presença de todos os picos característicos da estrutura da zeólita ZSM-5, quando comparados com o perfil padrão da estrutura MFI.

Figura 2 - Difratogramas das amostras sintetizadas pelo método conversão de gel seco.

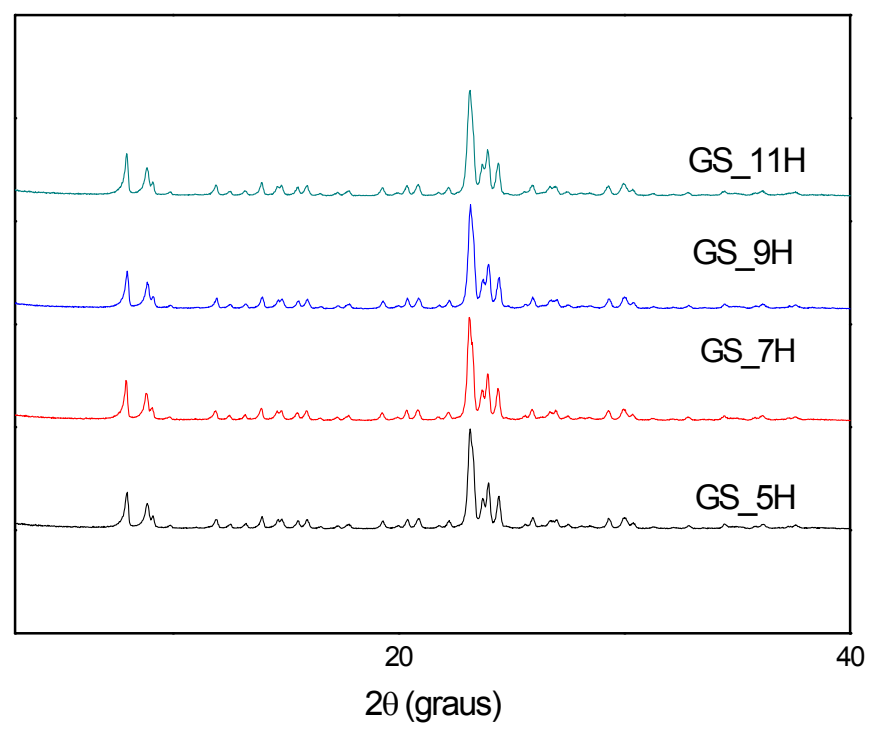


A partir dos difratogramas, foi possível obter o grau de cristalinidade do material. A Tabela 1 apresenta dados das amostras com percentuais de cristalinidade.

Tabela 1 - Propriedades dos sistemas (fluido-partícula).

\begin{tabular}{ccc}
\hline Amostra & Tempo (h) & Cristalinidade (\%) \\
\hline GS_5H & 5 & 93 \\
GS_7H & 7 & 93 \\
GS_9H & 9 & 95 \\
GS_11H & 11 & $100^{*}$ \\
\hline
\end{tabular}

*Amostra padrão.

\subsection{Análises térmicas}

A Figura 3 exibe os resultados das análises de TG/DTG realizado na amostra GS_11H. As curvas de percentual de perda de massa e DTG apresentam basicamente três eventos de perdas de massa.

Figura 3 - Curvas de TG/DTG da amostra GS_11H utilizada como padrão.

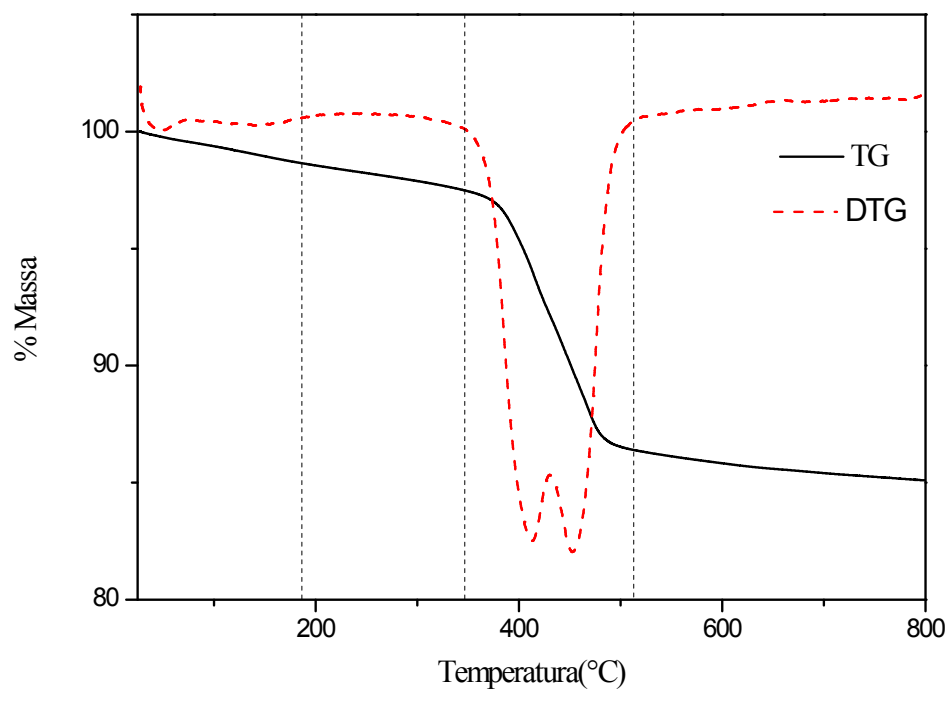

A partir da Figura 3, nota-se perdas de massa correspondente a primeira etapa, relacionada à eliminação água fisissorvida (evaporação de moléculas fisicamente adsorvidas, ou seja, grupos hidroxilas fracamente ligados às partículas) e resultado da remoção de água fortemente adsorvida. A segunda etapa na faixa de 350 a $510^{\circ} \mathrm{C}$, relacionada a decomposição oxidativa de íons amônio provenientes do agente direcionador. Por fim, como a remoção completa da água e da amônia aconteceu em torno de $550^{\circ} \mathrm{C}$ pode-se concluir que esta temperatura é ideal para calcinação das amostras de ZSM-5. 


\subsection{Adsorção de $\mathrm{N}_{2}$ (BET)}

A análise de adsorção de $\left(\mathrm{N}_{2}\right)$ a $77 \mathrm{~K}$ foi realizada na amostra GS_11H utilizada como padrão, na forma calcinada, com a finalidade de se obter informações sobre a área específica e a estrutura porosa do sólido. A isoterma de adsorção de nitrogênio da amostra está representada na Figura 4.

Figura 4 - Isotermas de Adsorção/Dessorção de $\mathrm{N}_{2}$ da amostra GS_11H.

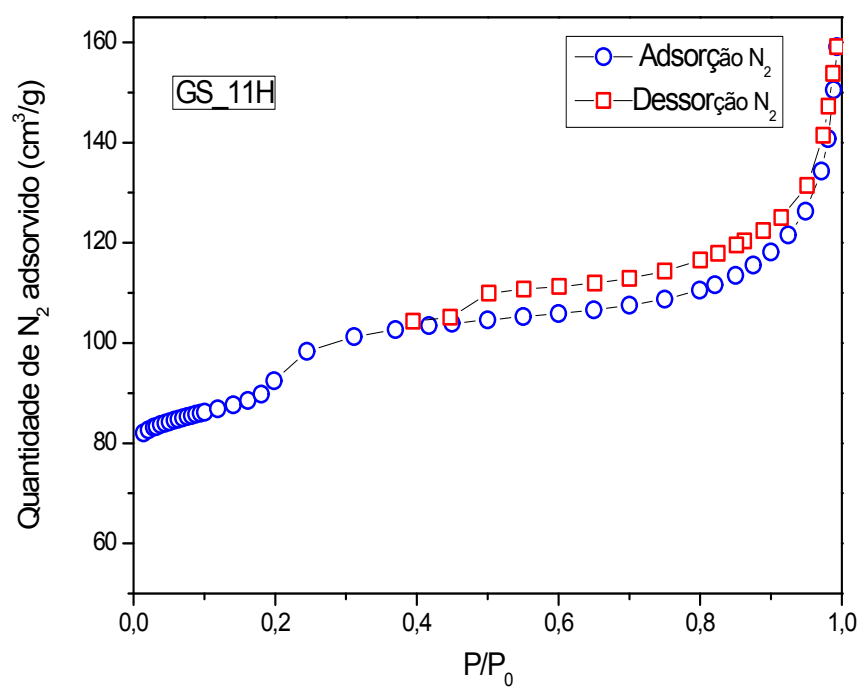

De acordo com a classificação da IUPAC, as isotermas da zeólita ZSM-5 são características de sólidos microporosos que podem ser classificadas como sendo do tipo I. No entanto, a amostra GS_11H, exibe um perfil de isoterma do tipo I modificada, com predominância de microporos e presença de mesoporos secundários, com um loop de histerese que pode ser enquadrado como sendo do tipo H4, que estaria relacionado com a formação de poros em forma de fenda (Figueiredo e Ribeiro, 1987).

Tabela 2 mostra a área específica, da amostra utilizada como padrão, que foi calculada pelo método BET.

Tabela 2 - Área específica da amostra padrão calculada pelo método BET

$$
\text { AMOSTRA ÁREA ESPECÍFICA }\left(\mathrm{m}^{2} \mathrm{~g}^{-1}\right)
$$

GS_11H 334

Através dos dados da Tabela 2, é possível afirmar que o valor da área específica calculada pelo método BET foi elevado, característicos dos sólidos microporosos, sendo condizentes com os encontrados na literatura, confirmando a cristalização da ZSM-5. 


\section{CONCLUSÕES}

No presente trabalho buscou-se avaliar a síntese por conversão de gel seco, assim como caracterizar as propriedades físico-químicas de zeólitas com estrutura ZSM-5, obtendo as seguintes conclusões:

As análises de difração de raios-X indicaram a presença de todos os picos característicos da estrutura da zeólita ZSM-5.

As curvas TG/DTG foram utilizadas para monitorar a remoção do direcionador dos poros da estrutura zeolítica e com indicação da temperatura adequada para calcinação dos materiais. A partir destes dados foi possível sugerir que a temperatura de $550^{\circ} \mathrm{C}$ é suficiente para remoção do direcionador estrutural.

Foi observado que a forma da isoterma de adsorção/dessorção de $\mathrm{N}_{2}$ apresentarou "loop" de histerese na dessorção a altas pressões parciais, que revela algum grau de mesoporosidade. E o valor da área específica calculada pelo método BET foi elevados, característicos dos sólidos microporosos.

A totalidade dos resultados obtidos permitiu concluir que a síntese pelo método de conversão de gel seco foi eficaz, comprovada pelas propriedades estrutura, cristalinidade, composição da rede, morfologia e porosidade, em termos comparativos com banco de dados apresentados pela IZA.

\section{REFERENCIAS}

CHEN, B.; HUANG, Y. Dry gel conversion synthesis of SAPO- and CoAPO- based molecular sieves by using structurally related preformed ALPO precursors as the starting materials. Micropor. Mater., v. 123, 71-77, 2009.

FIGUEIREDO, J. L.; RIBEIRO, F. R. Catálise heterogênea. $1^{\mathrm{a}}$ Ed. Fund. Calouste Gulbenkian: Lisboa, 1987.

GIANNETTO, G. P. Zeolitas - Características, propiedades y aplicaciones industriales. Editorial Innovación Tecnológica, Caracas, 2000. 170.

MASTERS,A.F.;MASCHMEYER,T.; Microporous Mesoporous Mater. 2011.

MATSUKATA, M.; OGURA, M.; OSAKI, T.; RAO, P. R. H. P.; NOMURA, M.; KIKUCHI E. Conversion of dry gel to microporous crystal in gas phase. Top. Catal., v. 9, 77-92, 1999. 